\title{
Pradimicin-resistance of Yeast is Caused by a Point Mutation of the Histidine-containing
}

\section{Phosphotransfer Protein Ypd1}

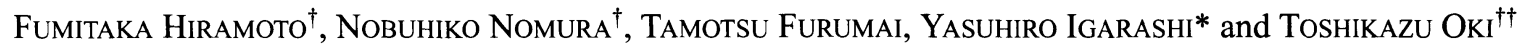 \\ Biotechnology Research Center, Toyama Prefectural University, \\ 5180, Kurokawa, Kosugi, Toyama 939-0398, Japan
}

(Received for publication August 4, 2003)

\begin{abstract}
Pradimicin is an antifungal antibiotic which induces apoptosis like cell death in the yeast Saccharomyces cerevisiae. Pradimicin-resistant mutants were isolated from the $S$. cerevisiae and the mutation points were analyzed. A point mutation of YPD1 that led to a substitution of the 74th glycine (Gly74) to cysteine (Cys) was identified in a mutant strain NH1. In $S$. cerevisiae, Ypd1 transfers a phosphoryl group from the sensor kinase Sln1 to the response regulator Ssk1 which regulates a downstream MAP kinase in response to hyperosmotic stress. Gly74 is located in a three-residue reverse turn domain that connects two $\alpha$-helices, one of which contains a histidine residue which is phosphorylated. In the reverse turn, glycine (relative position +10 to the active-site histidine) is highly conserved in Ypdl and other histidinecontaining phosphotransfer proteins. It was therefore suggested that the substitution of Gly 74 to Cys altered the Ypdl structure, which resulted in the resistance to pradimicin.
\end{abstract}

Apoptosis is a highly regulated process of programmed cell death and plays a central role in development and homeostasis of metazoan organisms. Its complex regulatory network in multicellular system has been studied using model organisms such as Dorosophila melanogasterbor and Caenorhabditis elegans ${ }^{1}$. Apoptosis had been assumed to be confined to multicellular organisms, because a suicide mechanism seemed useless for a unicellular organism like yeast. In fact, homologues of apoptosis-related proteins in metazoans were not found in the genome sequence of Saccharomyces cerevisiae. However, the expression of metazoan-derived apoptosis inducers such as Bax, caspase and p53 caused cell death in yeast, and Bcl-2, an apoptosis suppressor in animals, circumvented the Bax-induced cell death in yeast ${ }^{2 \sim 4)}$. It was also reported that the apoptotic morphological and molecular changes in yeast was induced by a point mutation of $C D C 48$ encoding a cell cycle protein ${ }^{5,6)}$.

Pradimicin is an antifungal antibiotic produced by actinomycetes (Fig. 1). It is a promising lead compound with high selectivity to fungi owing to its specific binding affinity to mannose residues in the cell surface mannan.
Previously, we reported that the apoptosis-like cell death was induced in yeast by pradimicin, associated with typical apoptotic markers such as DNA fragmentation and the accumulation of reactive oxygen species ${ }^{7}$. Noteworthily,

Fig. 1. Structure of pradimicins $\mathrm{A}, \mathrm{L}$ and $\mathrm{S}$.

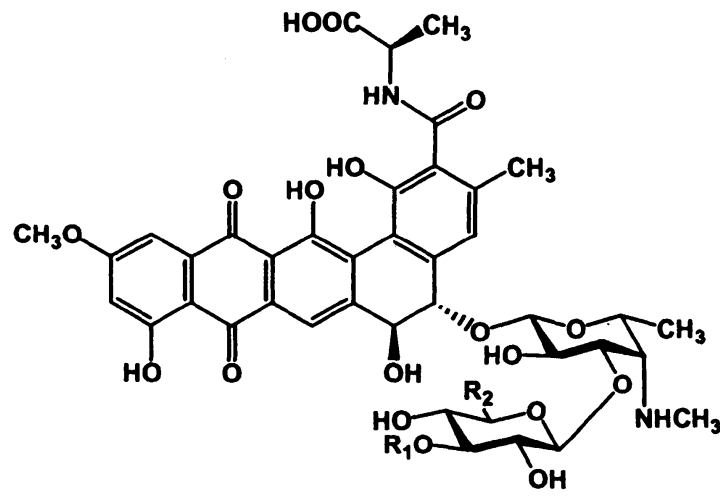

$$
\begin{aligned}
\text { Pradimicin A } & \mathrm{R}_{1}=\mathrm{R}_{2}=\mathrm{H} \\
\mathrm{L} & \mathrm{R}_{1}=\mathrm{H}, \mathrm{R}_{2}=\mathrm{CH}_{2} \mathrm{OH} \\
\text { S } & \mathrm{R}_{1}=\mathrm{SO}_{3} \mathrm{H}, \mathrm{R}_{2}=\mathrm{CH}_{2} \mathrm{OH}
\end{aligned}
$$

* Corresponding author: yas@pu-toyama.ac.jp

${ }^{\dagger}$ Present address: Toyama Chemical Co., Ltd., 4-2-1, Shimookui, Toyama 939-8508, Japan.

t† Present address: Tamagawa University Research Institute, 6-1-1, Tamagawa-gakuen, Machida, Tokyo 194-8610, Japan 
the pradimicin-induced cell death was prevented by an oxygen radical scavenger, $\mathrm{N}$-acetylcysteine, indicating that the manner of cell death resembles apoptosis in metazoans ${ }^{7}$. To elucidate the target site and fungicidal mechanism of pradimicin, we isolated pradimicin-resistant mutants of S. cerevisiae and analyzed their mutation points. We report herein that the function of Ypd1, a histidinecontaining phosphotransfer protein in yeast osmosensing system, is associated with the fungicidal action of pradimicin.

\section{Materials and Methods}

\section{Compounds}

Pradimicins $\mathrm{A}$ and $\mathrm{S}$ were isolated from the fermentation broth of actinomycetes sp. TP-A0016 and TP-A0123, respectively ${ }^{8}$. Pradimicin $\mathrm{L}$ was prepared from pradimicin $\mathrm{S}$ by acid hydrolysis ${ }^{9}$. Amphotericin B and pyrrolnitrin were purchased from Sigma Chemical Co. Micafungin was purchased from Fujisawa Pharmaceutical Co., Ltd., fluconazole from Pfizer Inc. and miconazole from Mochida Pharmaceutical Co., Ltd.

\section{Strains and Media}

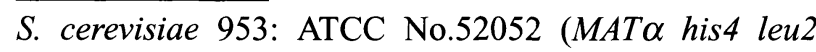

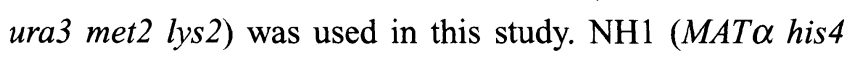
leu 2 ura 3 met 2 lys2) is a pradimicin-resistant mutant strain derived from the parent strain, $S$. cerevisiae 953. S. cerevisiae KYC291: IFO10482 (MATa his4 gal2) and $S$. cerevisiae YNN140: IFO10151 (MATa ura3 trpl his3 ade2) were used for the backcross experiment. Escherichia coli XL1-blue $\mathrm{MRF}^{\prime}: \Delta$ (mcrA) 183, $\Delta$ (mcrCB-hsdSMR-mrr) 173 endA1, supE44, thi-1, recAl, gyrA96, relA1, lac [ $\mathrm{F}^{\prime}$, proAB, lacl $\left.^{q} Z \Delta M 15, \operatorname{Tn} 10\left(\mathrm{Tet}^{\mathrm{I}}\right)\right]$ was used in this study.

YPD agar was consisted of yeast extract $1 \%$ (Difco), peptone $2 \%$ (Difco), dextrose $2 \%$ and agar $1.5 \%$ and SD agar yeast nitrogen-base $0.67 \%$ (Difco), dextrose $2 \%$ and agar $1.5 \%$ as a minimal medium, to which the appropriate supplements were added.

\section{Isolation of Pradimicin-resistant Mutants}

For the spontaneous mutation, S. cerevisiae 953 cells from the log-phase culture were plated directly on SD agar. For the chemical mutation, cells treated in the culture containing ethyl methanesulfonate (survival rate $30 \%$ ) were plated. Approximately $1 \times 10^{7}$ cells were plated on a SD medium plate supplemented with $50 \mu \mathrm{g} / \mathrm{ml}$ of pradimicin A for each mutagenesis. The susceptibility of the clones to pradimicin was examined and the mutants for further study were selected. Complementation groups were classified by crossing individual mutants and by testing the resistance of the corresponding diploids to pradimicin. When required, the diploids were sporulated and tetrad analysis was carried out and the mutants were backcrossed twice to ensure that their phenotypes were derived from a single gene by standard method ${ }^{10)}$.

\section{Susceptibility to Antifungal Agents}

The $\mathrm{IC}_{75}$ of pradimicins $\mathrm{A}, \mathrm{L}$ and $\mathrm{S}$ and other antifungal agents (amphotericin B, pyrrolnitrin, fluconazole, miconazole and micafungin) were determined by the microdilution method using SD broth supplemented with leucine, histidine, methionine, lysine and uracil (each $20 \mathrm{mg} /$ liter) on 96-well microplates. Yeast cells in the exponential growth were washed and resuspended in fresh SD broth and inoculated at a final concentration of $10^{4}$ cells $/ \mathrm{ml}$. After the incubation at $27^{\circ} \mathrm{C}$ for 84 hours, the $\mathrm{IC}_{75}$ was determined as the concentration of agents that inhibited $75 \%$ of the growth comparing with the control.

\section{Genetic Manipulation}

Genetic manipulation of E. coli and S. cerevisiae was conducted according to the standard methods ${ }^{10,11)}$. S. cerevisiae genomic library (ATCC 37415$)$ was used. DNA sequencing was carried out on an automated DNA sequencer (ALFred DNA sequencer, Pharmacia Biotech).

\section{Cloning of YPD1 Region from Strain NH1}

The YPDI region of strain NH1 was obtained by PCR using 5'-TTGAGGATCCACTGACGTGTAA-3' and 5'CTGTTACACTGCAGTATTTG- $3^{\prime}$ as primers and the chromosomal DNA of strain NH1 as a template. The PCR was carried out on a Perkin-Elmer Gene Amp2400 Thermocycler with the following parameters: $94^{\circ} \mathrm{C}$ for 3 minutes, followed by 25 cycles with three identical steps, $94^{\circ} \mathrm{C}$ for 1 minute, $55^{\circ} \mathrm{C}$ for 1 minute and $72^{\circ} \mathrm{C}$ for 1 minute, and finally $72^{\circ} \mathrm{C}$ for 3 minutes. The gene amplified by PCR was cloned into pUC18, which was designated pYPD1.

\section{Transformation with ypd1-URA3 Cassette}

The plasmid pYPD1 was digested by $B s p 1407 \mathrm{I}$ located in the non-coding region in the downstream of $Y P D 1$, filled in with Klenow fragment (Takara Bio Inc.) at the noncoding region in the downstream of YPDI and ligated with URA3 cassette which was obtained from pYE-Ura3 (TOYOBO Co., Ltd.) by digesting with EcoRI and HindIII and filling. ypdl-URA3 cassette was transformed to the wild type strain and the clones were selected on a SD agar 
Table 1. Characterization of pradimicin-resistant mutants.

\begin{tabular}{lcccc}
\hline & \multicolumn{3}{c}{ pradimicin $(\mu \mathrm{g} / \mathrm{ml})^{1)}$} & t.s. $^{2)}$ \\
\cline { 2 - 5 } & 6.25 & 25 & 100 & $37^{\circ} \mathrm{C}$ \\
\hline S.c. 953 & - & - & - & + \\
NH1 (3)* & + & + & + & - \\
NH2 (5) & + & + & + & + \\
NH3 (4) & + & + & + & + \\
NH4 (1) & + & + & - & + \\
NH5 (2) & + & + & - & + \\
NH6 (4) & + & - & - & + \\
\hline
\end{tabular}

1) $\mathrm{SD}$ agar, (-) not grown, $(+)$ grown

2) temperature sensitivity, $37^{\circ} \mathrm{C}$ on YPD agar, inoculum size $10^{3}$ cells /spot, $(-)$ not grown, $(+)$ grown

* The number of phenotypically and genotypically same mutants is indicated in parenthesis.

plate which did not contain uracil.

\section{Results}

Isolation of Pradimicin-resistant $S$. cerevisiae Mutants

One-hundred and twenty pradimicin-resistant mutants were isolated on SD agar plates containing $50 \mu \mathrm{g} / \mathrm{ml}$ pradimicin A. The MIC of pradimicin A against the resistant mutants ranged from 12.5 to $>100 \mu \mathrm{g} / \mathrm{ml}$. The mutation in pradimicin-resistance was indicated recessive by the observation that all the diploids were susceptible to pradimicin A as same as the parent strain. Among 120 mutant strains, 19 strains could be classified into six complementation groups (NH1 NH6, Table 1), based on the phenotypic (pradimicin-resistance, temperature sensitivity) and genotypic (complementation test) grouping.

\section{Phenotype of Strain NH1}

Summarized in Table 2 is the susceptibility of strain NH1 to antifungal agents. Strain NH1 showed resistance to three pradimicin congeners whereas it was more susceptible to azoles than the wild type of $S$. cerevisiae. The susceptibility to other agents was almost equal to that of the wild strain. The temperature sensitivity of strain NH1 was indicated by the growth inhibition at $37^{\circ} \mathrm{C}$ (Fig. 2).
Table 2. Susceptibility of pradimicin-resistant mutant strain NH1 to antifungal agents.

\begin{tabular}{lcc}
\hline & \multicolumn{2}{c}{$\mathrm{IC}_{75}(\mu \mathrm{g} / \mathrm{ml})^{1)}$} \\
\cline { 2 - 3 } Agents & wild type & $\mathrm{NH1}$ \\
\hline Pradimicin A & 6.25 & $>100$ \\
Pradimicin L & 3.13 & $>100$ \\
Pradimicin S & 6.25 & 50 \\
Amphotericin B & 0.20 & 0.39 \\
Pyrrolnitrin & 25 & 12.5 \\
Fluconazole & $>100$ & 25 \\
Miconazole & 1.56 & 0.20 \\
Micafungin & 0.39 & 0.20 \\
\hline 1) Broth dilution method, inoculum size & $10^{4}$ cells $/ \mathrm{ml}$ \\
SD medium. & \multicolumn{2}{l}{}
\end{tabular}

Fig. 2. Complementation of the phenotype of strain NH1 by YPD1.

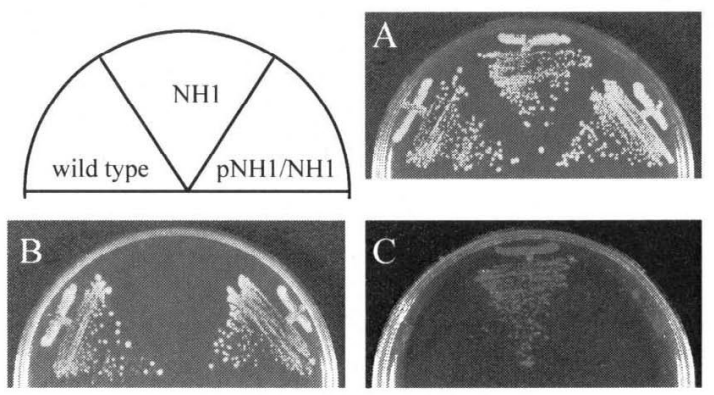

YPD1 was carried on plasmid pNH1.

(A) YPD medium, $26^{\circ} \mathrm{C}$, (B) YPD medium, $37^{\circ} \mathrm{C}$,

(C) $\mathrm{SD}$ medium with $6.25 \mu \mathrm{g} / \mathrm{ml}$ pradimicin $\mathrm{A}, 26^{\circ} \mathrm{C}$.

\section{Isolation of a Single Copy Suppressor for the Mutation in Strain NH1}

The mutation in strain NH1 was recessive and therefore strain NH1 was transformed with the $S$. cerevisiae wild type genomic library. The transformants applied on SD agar were incubated at $37^{\circ} \mathrm{C}$ to select the transformant carrying the gene complementary to temperature sensitivity since the phenotype was considered associated with pradimicin-resistance. During the incubation emerged a colony which regained the temperature tolerance and the sensitivity to pradimicin.

The plasmid recovered from the clone contained a $7 \mathrm{~kb}$ 
Fig. 3. Pradimicin-resistant phenotype by transformation of $y p d 1 \mathrm{G} 74 \mathrm{C}$ to wild type strain.

(a)

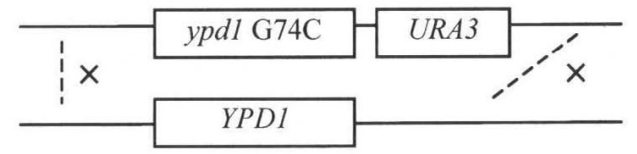

(b)
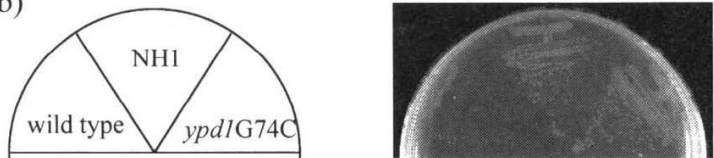

(a) The cassette containing ypdlG74C and URA3 was transformed in the wild type strain.

(b) Growth of wild type (S. c. 953), NH1 and ypd1G74C on SD agar plate supplemented with $6.25 \mu \mathrm{g} / \mathrm{ml}$ of pradimicin A after incubation at $26^{\circ} \mathrm{C}$ for 3 days.

genomic DNA fragment in which were included four open reading frames, $P H O 13, Y P D 1, G Y P 7$ and YDL233. Based on the restriction enzyme map, partially deleted plasmids of this DNA fragment were constructed and transformed to strain NH1. The phenotype of strain NH1 was complemented by the vector of $2 \mathrm{~kb}$ Pst I-HpaI fragment containing an ORF of YPDI (Fig. 3).

\section{Analysis of YPD1 Mutation Point in Strain NH1}

YPD1 region of strain $\mathrm{NH} 1$ was amplified by PCR, sequenced and compared with that of the wild type strain. The 220th guanine of YPDI was substituted with thymidine, which resulted in the substitution of the 74th amino acid glycine to cysteine in Ypd1. Furthermore, the ypd1 G74C-URA3 cassette was transformed to the wild strain. The transformation was confirmed by amplifying the cassette gene by PCR and sequencing the transformed YPDI region. The strain replaced with ypdl G74C showed pradimicin-resistance (Fig. 3) and other phenotypes identical with those of strain NH1 (data not shown).

\section{Discussion}

In $S$. cerevisiae, osmolarity is regulated in part through a multistep phosphoryl transfer reactions (Fig. 4) ${ }^{12)}$. A key component in this phosphotransfer system is Ypdl which functions as a histidine-containing phosphotransfer. Ypd1 mediates phosphoryl group transfer from the
Fig. 4. Schematic diagram of osmosensing signal transduction pathway in $S$. cerevisiae.

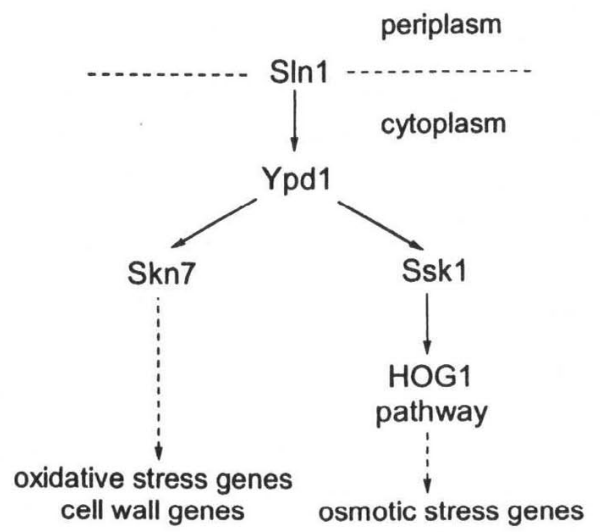

transmembrane osmosensor kinase $\operatorname{Sln} 1$ to a response regulator protein $\mathrm{Ssk} 1^{13)}$. Under normal osmotic conditions, Ssk1 is maintained in a phosphorylated inactive state. Ssk1 becomes dephosphorylated active form under hyperosmotic stress conditions, which allows Ssk1 to activate the downstream HOG1-MAP kinase cascade which ultimately results in elevated levels of intracellular glycerol. In addition, Ypd1 mediates phosphoryl transfer from Sln1 to $\mathrm{Skn} 7$, another response regulator protein which functions in response to oxidative stress and cell wall damage ${ }^{14)}$.

X-Ray crystallographic analysis revealed that a fourhelix bundle comprises the core structure of $\mathrm{Ypd} 1$ and two helices, one of which contains the histidine that is phosphorylated, are joined by a conserved three-residue reverse turn ${ }^{15)}$. Among the three residues, glycine relatively positioned at +10 to the active-site histidine is highly conserved in Ypdl and other proteins that possess histidine-containing phosphotransfer activity. The glycine structure was suggested important in Ypdl protein folding by the heterologous expression of $y p d l$ G74A mutant in $E$. coli, which resulted in the aggregation and insolubility in the cell ${ }^{16}$. We here proved that a point mutation leading to the substitution of the 74th glycine in the reverse turn to cysteine in Ypd1 resulted in the complete resistance of $S$. cerevisiae to pradimicin. The high conservation of the glycine residue indicates its possible role in maintaining the function of Ypd1. Although the functional role of Ypd1 in fungicidal action of pradimicin is still unknown, it is clear that the ypdl G74C mutation prevents the apoptotic cell death of $S$. cerevisiae induced by pradimicin. The $y p d l$ G74C mutant showed hypersensitivity to high osmolarity 
(e.g. $1.4 \mathrm{M} \mathrm{NaCl}$, results not shown), indicating that its HOG1 pathway is not functional. The mutant also showed temperature sensitivity that is likely induced by the alteration of the Ypdl structure and function which affects the expression of genes responsible for stress response (Fig. 4).

Ypd1 plays a central role in the yeast osomosensing signal transduction system. Analysis of the involvement of other signal transduction proteins and the mutation point of the remaining pradimicin-resistant mutants are now in progress.

\section{References}

1) STELleR, H.: Mechanisms and genes of cellular suicide. Science 267: 1445 1449, 1995

2) Sato, T.; M. Hanada, S. Bodrug, S. Irie, N. Iwama, L. H. Boise, C. B. Thompson, E. Golemis, L. Fong \& H. G. WANG: Interactions among members of the Bcl-2 protein family analyzed with a yeast two-hybrid system. Proc. Natl. Acad. Sci. USA 91: 9238 9242, 1994

3) Kang, J. J.; M. D. Schaber, S. M. SRinivasula, E. S. Alnemri, G. Litwack, D. J. Hall \& M. A. BJornsti: Cascades of mammalian caspase activation in the yeast Saccharomyces cerevisiae. J. Biol. Chem. 274: 3189 3198, 1999

4) Nigro, J. M.; R. Sikorski, S. I. ReEd \& B. Vogelstein: Human p53 and CDC2Hs genes combine to inhibit the proliferation of Saccharomyces cerevisiae. Mol. Cell. Biol. 12: 1357 1365, 1992

5) MadeO, F.; E. FröHlich \& K-U. FRöHlich: A yeast mutant showing diagnostic markers of early and late apoptosis. J. Cell. Biol. 139: 729 734, 1997

6) Madeo, F;; E. Fröhlich, M. Ligr, M. Grey, S. J. SigRist, D. H. WOLF \& K-U. FRöHLICH: Oxygen stress: a regulator of apoptosis in yeast. J. Cell. Biol. 45: 757 767, 1999

7) Hiramoto, F.; N. Nomura, T. Furumai, T. OKi \& Y.
IGARASHI: Apoptosis-like cell death of Saccharomyces cerevisiae induced by a mannose-binding antifungal antibiotic, pradimicin. J. Antibiotics 56: 768 772, 2003

8) Dairi, T.; Y. Hamano, Y. Igarashi, T. Furumai \& T. OKI: Cloning and nucleotide sequence of the putative polyketide synthase genes for pradimicin biosynthesis from Actinomadura hibisca. Biosci. Biotech. Biochem. 61: 1445 1453, 1997

9) Saitoh, K.; T. Tsuno, M. Kakushima, M. Hatori, T. Furumai \& T. OKI: Pradimicin S, a new pradimicin analog. II. Isolation and structure elucidation. J. Antibiotics 46: 406 411, 1993

10) Rose, M. D.; F. Winston \& P. Hieter: Methods in yeast genetics: a laboratory course manual. Cold Spring Harbor Laboratory Press, New York, 1990

11) SAMBrooK, J.; E. F. FriTsch \& T. MANiatis: Molecular cloning: a laboratory manual (2nd ed.) Cold Spring Harbor Laboratory Press, New York, 1989

12) HohmanN, S.: Osmotic stress signaling and osmoadaptation in yeasts. Microbiol. Mol. Biol. Rev. 66: 300 372, 2002

13) Posas, F.; S. M. Wurgler-Murphy, T. Maeda, E. A. Witten, T. C. Thai \& H. SAito: Yeast HOG1 MAP kinase cascade is regulated by a multistep phosphorelay mechanism in the SLN1-YPD1-SSK1 "two-component" osmosensor. Cell 86: 865 875, 1996

14) Li, S.; A. Ault, C. L. Malone, D. Raitt, S. Dean, L. H. Jhonston, R. J. Deschenes \& J. S. FAssler: The yeast histidine protein kinase, $\operatorname{Sin} 1 \mathrm{p}$, mediates phosphotransfer to two response regulators, Ssk1p and $\mathrm{Skn} 7 \mathrm{p}$. EMBO J. 17: $6952 \sim 6962,1998$

15) $\mathrm{XU}, \mathrm{Q}$. \& A. H. West: Conservation of structure and function among histidine-containing phosphotransfer (HPt) domains as revealed by the crystal structure of YPD1. J. Mol. Biol. 292: 1039 1050, 1999

16) JANIAK-SPENS, F. \& A. H. WeST: Functional roles of conserved amino acid residues surrounding the phosphorylatable histidine of the yeast phosphorelay protein YPD1. Mol. Biol. 37: 136 144, 2000 